\section{Late Congenital Syphilis: A Rarity but Reality}

\section{Ishaq Ghauri, Imadud-Deen*, Ashok Kumar, Ajeet Kumar and Salma Razzak}

Department of Medicine, Jinnah Medical College Hospital, Karachi, Pakistan

\begin{abstract}
Introduction: Congenital syphilis is an infectious disease caused by a spirochete Treponema Pallidum. It is transmitted transplacentlly from an infected mother to the fetus. Congenital syphilis is a preventable disease and its presence reflects a failure of antenatal care system. Its presence emphasizes on the development of effective screening and treatment programs. Congenital syphilis may present as early, late or stigmata.

Case presentation: Here we present a case of a 13yr old male child who came from interior Sindh, who presented to us with stigmata of syphilis, but unfortunately remained undiagnosed.

Conclusion: The correct diagnosis of our patient was based on clinical suspicion, appropriate lab tests, and deliberation of the differential diagnosis. The management of our patient depended on the diagnosis of correct cause of the complaints.

Keywords: Sexually transmitted disease; Syphilis; Treponema
\end{abstract} Pallidum

\section{Introduction}

Congenital syphilis is a rare and severe infectious disease often with serious and disabling consequences. It is a multisystem infectious disease caused by a spirochete Treponema pallidum and is transmitted to the fetus via placenta. Although this disease is preventable when mothers with syphilis are treated early in pregnancy [1], but its continued occurrence shows a failure of the basic systems of antenatal care and control of sexually transmitted diseases [2]. Although the antenatal screening for syphilis is cost effective and cost beneficial both in developed and developing countries [3], but it has been seen that the rate of congenital syphilis is declining in developed countries, while a significant increase has been observed in developing countries $[4,5]$. Congenital syphilis is classified as early (i.e., birth through 2 nd year of life), late (after 2 Years of age), stigmata (scars and deformities which are the consequences of early and late syphilis). Late congenital syphilis is a rare entity and it should be considered as an avoidable complication of congenital syphilis because

*Corresponding author: Imadud-Deen, Department of Medicine, Jinnah Medical College Hospital, Karachi, Pakistan, Tel: +92 3333950852; E-mail: imaduddeen@live.com

Citation: Ghauri MI, Imadud-Deen, Kumar A, Kumar A, Razzak S (2016) Late Congenital Syphilis: A Rarity but Reality. J Clin Stud Med Case Rep 3: 027.

Received: November 12, 2015; Accepted: December 29, 2015; Published: January 14, 2016 it is almost completely prevented when early congenital syphilis, asymptomatic or symptomatic is treated and careful serologic follow-up is carried out. Approximately $66 \%$ of infected infants from congenital syphilis are asymptomatic at the time of birth and are identified only by routine prenatal screening. Untreated syphilis during pregnancy has a transmission rate of approximately $100 \%$ [6], and $40 \%$ of infected fetuses die in intrauterine prenatal period while $60 \%$ of surviving infants will have late manifestations if left untreated [7].

World Health Organization (WHO) estimates that worldwide two million pregnant women get infected with syphilis every year. In pregnant women, the incidence ranges from 1.5\% in Europe to $3.9 \%$ in parts of the United States and 1.48\% in South East Asia [8]. Sadly no data is present on the prevalence of syphilis in pregnant women in Pakistan.

The leading factor for the continued high prevalence of congenital syphilis globally, is the lack of adequate prenatal care [9]. Congenital syphilis poses a great challenge for the clinicians. More than $50 \%$ of live born affected infants may be asymptomatic and many of them are often not reported [10].

The main purpose of reporting this case is to emphasize on the importance of antenatal screening of syphilis and diagnose the patient, soon before the onset of certain disabling signs and symptoms. Here we report a case of a 13 year old boy with late congenital syphilis presented to us with classical stigmata, who remained undiagnosed for many years.

\section{Case Presentation}

13 year old, mentally retarded, male, came from a small town of Sindh, who presented in outpatient department with complaints of deformities in both hands since 9 years, blurring of vision since 6 years and pain in knee joints since 2 years. He started having restricted hand movements 4 years after birth. Initially it was mild, later on, after approximately two years, it got severe along with onset of pain. He was taken to multiple doctors where his tests were done including serum rheumatoid factor and serum anti-nuclear antibody which turned out to be negative so patient was given analgesics and physiotherapy was advised. He also had on and off pain in his ankle joint that was initially moderate in intensity and progressed to his knee joints which resulted in difficulty in walking. There was progressive loss of vision in the right eye resulting in blindness of right eye. Patient had multiple visits to general physicians but no diagnosis was made and finally he was referred to us.

No significant family history was present. Mother had no antenatal visits and child was delivered at home. Patient was the youngest of 11 children, the rest are all healthy. Patient was conscious and oriented with a low IQ. His examination from head to toe showed conjunctival pallor, but no jaundice or cyanosis. Right eye showed multiple opacities in cornea. While left was normal. His nasal bridge was depressed with a 'saddle' shape deformity. His upper incisors were peg shaped classically Hutchinson's teeth and perioral fissures were also present. His mandible was also protruding giving the appearance of "bull dog facies". His hearing test was also done that showed sensory 
neural hearing loss in right ear. On lower limb examination, anterior bowing and thickening of tibia i.e., saber shins was present with atrophic scarred skin over both the shins which at places showed punched out ulcerations and crusts (Figure 1). Generalized lymphadenopathy was present involving the cervical, axillary, epitrochlear and inguinal region.

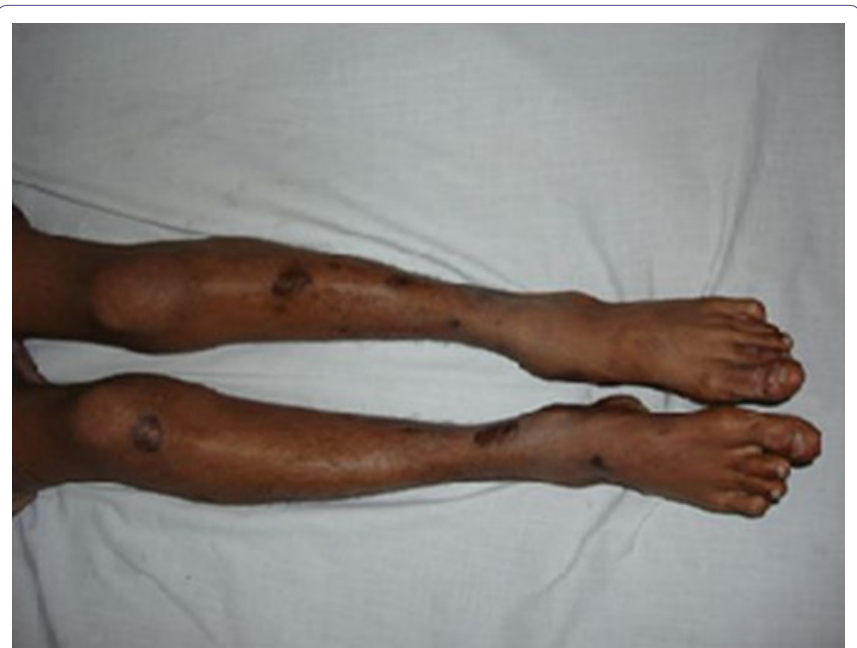

Figure 1: Skin ulcers can be seen on both legs along with saber shins.

Musculoskeletal examination revealed that both hands were deformed especially involving the index finger and little finger which were completely flexed and stiff, associated with nodular swelling in distal interphalangeal joints of these fingers (Figure 2). Both knee joints were swollen and inflamed. On examination of back we found flaring of scapulas (Figure 3). On the basis of history and examination it was suspected that the patient had congenital syphilis.

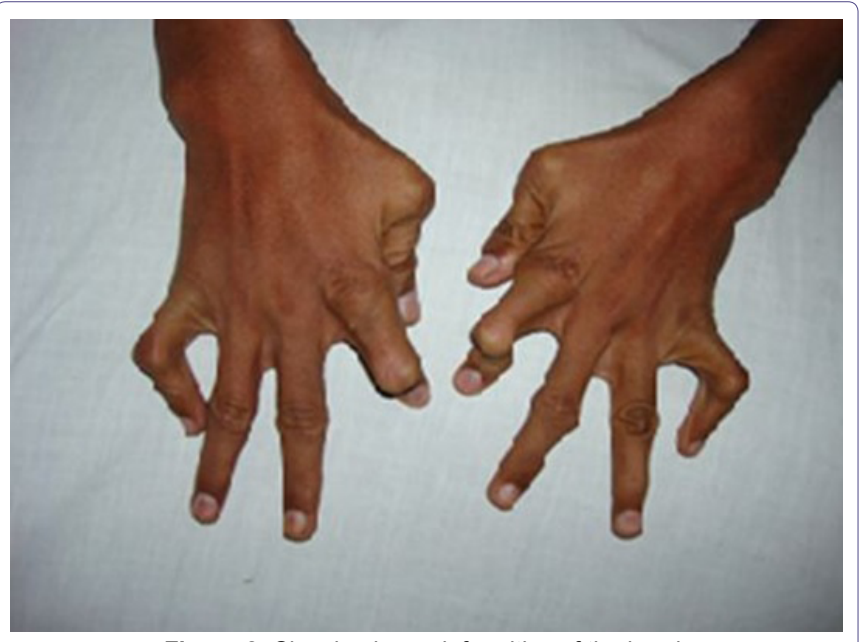

Figure 2: Showing bone deformities of the hand.

Routine investigations were normal. VDRL (Venereal Disease Research Laboratory) was reactive in dilution of 1:16 and TPHA (T. Pallidum Haemagglutination Assay) was also positive. FTA-BS was not performed because the patient's family had affordability issues as the test is expensive and dark field microscopy of the lesions was not done because of its unavailability in our hospital. Radiologic examination of both legs showed irregular periosteum and cortex at anterior and medial margin of midshaft of tibia and hands showed flexed index finger and little finger bilaterally (Figure 4). CSF examination was not done as consent was not given by patient's parents. Diagnosis of late congenital syphilis was made on the basis of

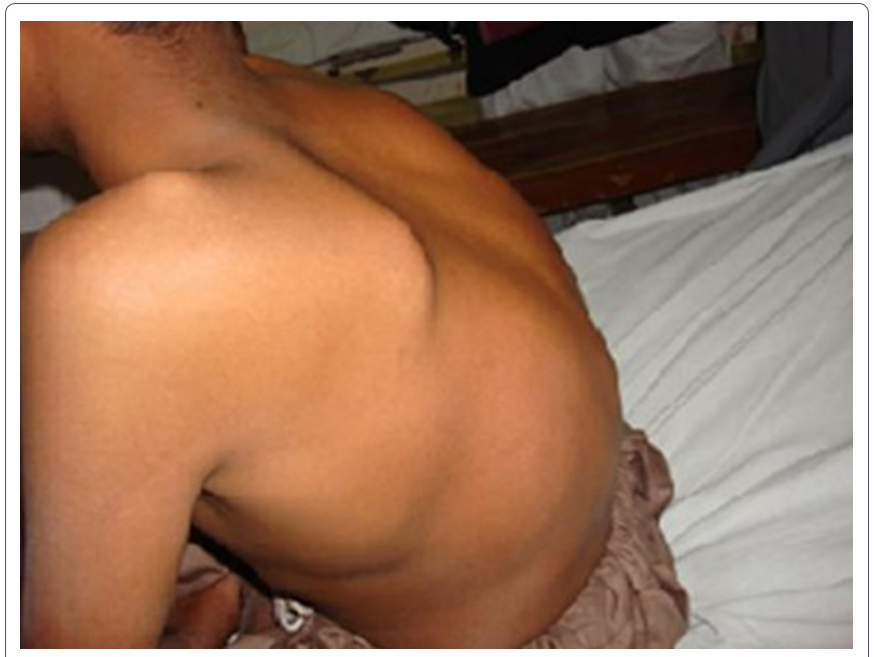

Figure 3: Shows the characteristic flaring of scapula in this patient.

physical examination and serological testing and the child was started on aqueous crystalline penicllin $\mathrm{G}$ as per CDC guidelines, administered as 50,000 units/ $/ \mathrm{kg} /$ dose intravenously, every 12 hours during the first 7 days of life and every 8 hours thereafter for a total of 10 days. The patient was followed up every month for a period of 6 months. His non treponemal antibody titers began to decline to $1: 8$ after a period of 2 months and the child was seronegative after 6 months.

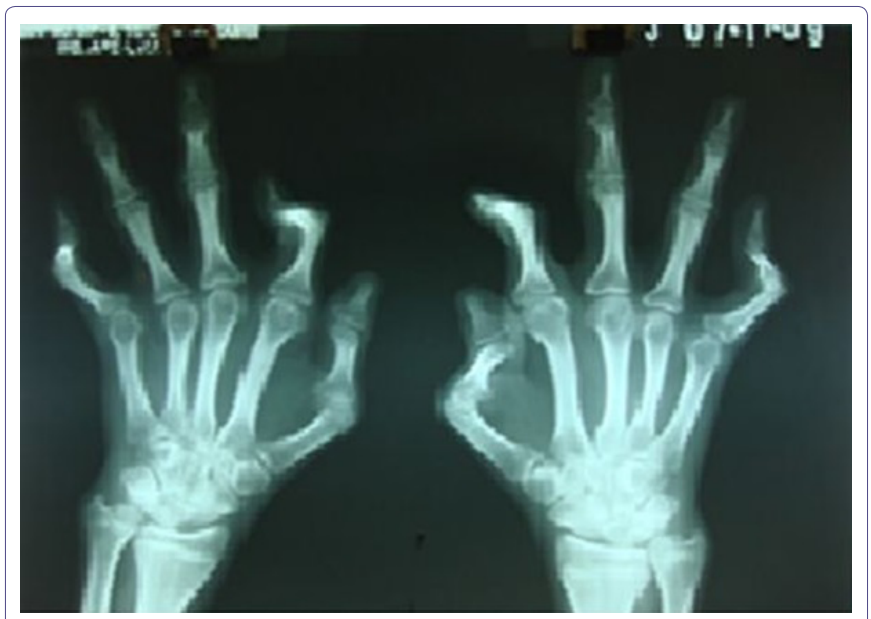

Figure 4: Shows radiological evidence of abnormalities present in both hands.

\section{Discussion}

Congenital syphilis is an infectious illness which still represents a financial and emotional burden in developing countries. The risks of vertical transmission and fetal disease depend upon the stage of syphilis in mother during pregnancy [11]. The risk of fetal loss and congenital syphilis drops slightly in early latent stage and decreases to $10 \%$ in late latent stage respectively $[12,13]$. Congenital syphilis is more likely to occur where mothers have poor antenatal care, that is when they have late, few or no antenatal visits. A recent data from WHO states that only $68 \%$ of women in developing countries receive antenatal care and among them half do not attend antenatal clinics until after first trimester [14].

Late congenital syphilis is usually characterized by Hutchinson's triad of interstitial keratitis, Hutchinson's incisors, and 8th cranial nerve deafness. Other presentations include mental retardation, 
hydrocephalus, mulberry molars, clutton's joints, saber shins. Late congenital syphilis diagnosed by non treponemal (VDRL and RPR) and treponemal (FTA-ABS, MHA-TP, and TPHA) tests. Non treponemal antibodies are $\operatorname{IgG}$ which transmitted placentally, makes interpretation of positive results difficult [15]. Therefore, it is necessary to compare the infant's titers with maternal serological titers using the same test. While a titer that is fourfold higher in the infant is accepted as significant, any increased titer should raise suspicion [16]. Presence of IgM in infant indicates active infection because IgM do not cross placenta, and this can be detected by FTA-ABS treponemal test. According to 1 study this test has a sensitivity in $88 \%$ of children with clinical features of Syphilis [17]. Definitive diagnosis is established by identifying organisms in body fluid or tissue by dark field microscopy, immunofluorescence, or histological examination. In our case 13 year old child presented with classical stigmata and positive VDRL and TPHA test enable us to label this case as late congenital syphilis.

The CDC recommends that any child with late congenital syphilis be treated with aqueous crystalline penicillin G 50,000 units/kg intravenously 4 to 6 hourly for 10 days.

According to WHO all pregnant patients should be screened for syphilis in the first trimester and again in late pregnancy [18]. Going by this recommendation had VDRL testing been performed in this mother in late pregnancy, she could have been diagnosed and treated adequately along with prevention of congenital syphilis in the newborn as she had no antenatal care. The patient came to us late because they lived in a remote area with lack of proper health care facility and due to illiteracy about the extent and nature of the disease. Therefore it can safely be said that vigilant screening prenatally at delivery and an adequate follow-up are critical to reduce the incidence of congenital syphilis.

\section{Conclusion}

For successful reduction of the burden of congenital syphilis, there should be early identification of infection in pregnant women via screening; adequate treatment of the woman, and identification and treatment of infected partners; modification of high-risk behavior; and promotion of access to and use of health care, particularly early antenatal care. Briefly, countries need to reexamine their current policies regarding the antenatal health care systems and steps must be taken to overcome all administrative and cultural barriers.

\section{Consent}

Written informed consent was obtained from the patient's legal guardian(s) for publication of this case report and any accompanying images. A copy of the written consent is available for review by the Editor-in-Chief of this journal.

\section{Competing Interests}

The authors declare that they have no competing interests.

\section{Authors' Contribution}

IG carried out the necessary tests for the diagnosis of stage of disease. AK conceived of the study .ASK interpreted all the results of the diagnostic tests and did the necessary examinations. SR conceived of the study. ID took the history of the patient and helped to draft the manuscript.

\section{Acknowledgements}

Authors do not have any commercial associations and no financial support has taken for this case report.

\section{References}

1. Stoll BJ (1994) Congenital syphilis: evaluation and management of neonates born to mothers with reactive serologic tests for syphilis. Pediatr Infect Dis J 13: $845-852$.

2. Holmes KK, Levine R, Weaver M (2004) Effectiveness of condoms in preventing sexually transmitted infections. Bull World Health Organ 82: 454-461.

3. Walker DG, Walker GJ (2002) Forgotten but not gone: the continuing scourge of congenital syphilis. Lancet Infect Dis 2: 432-436.

4. Gurlek A, Alaybeyoglu NY, Demir CY, Aydoğan H, Coban K, et al. (2005) The continuing scourge of congenital syphilis in 21 st century: a case report. Int $\mathrm{J}$ Pediatr Otorhinolaryngol 69: 1117-1121.

5. Chaudhary M, Kashyap B, Bhalla P (2007) Congenital syphilis, still a reality in 21st century: a case report. J Med Case Rep 1: 90.

6. World Health Organization (2009) Regional strategy for the elimination of congenital syphilis. Regional Office for South East Asia, World Health Organization, New Delhi, India.

7. Larsen SA, Steiner BM, Rudolph AH (1995) Laboratory diagnosis and interpretation of tests for syphilis. Clin Microbiol Rev 8: 1-21.

8. Patterson MJ, Davies HD (2012) Syphilis (Treponema pallidum). In: Kliegman RM, Stanton BF, Schor NF, St. Geme III JW, Behrman RE (eds.). Nelson Textbook of pediatrics. (19thedn), Elsevier, Philadelphia. Pg no: 1016.

9. Larkin JA, Lit L, Toney J, Haley JA (1998) Recognizing and treating syphilis in pregnancy. Medscape Womens Health 3: 5.

10. Humphrey MD, Bradford DL (1996) Congenital syphilis: still a reality in 1996. Med J Aust 165: 382-385.

11. World Health Organization, Department of Reproductive Health and Research (2005) Eliminating congenital syphilis. A global health priority. Sexual and reproductive health, World Health Organization, Geneva, Switzerland.

12. Fiumara NJ, Fleming WL, Downing JG, Good FL (1952) The incidence of prenatal syphilis at the Boston City Hospital. N Engl J Med 247: 48-52.

13. Li Y, Gonik B (2006) Is congenital syphilis really congenital syphilis? Infect Dis Obstet Gynecol 2006: 81629.

14. WHO, UNICEF (2003) Antenatal care in developing countries - Promises, achievements and missed opportunities. World Health Organization, Geneva, Switzerland.

15. Chang SN, Chung KY, Lee MG, Lee JB (1995) Seroreversion of the serological tests for syphilis in the newborns born to treated syphilitic mothers. Genitourin Med 71: 68-70.

16. Sánchez PJ, Wendel GD Jr, Grimprel E, Goldberg M, Hall M, et al. (1993) Evaluation of molecular methodologies and rabbit infectivity testing for the diagnosis of congenital syphilis and neonatal central nervous system invasion by Treponema pallidum. J Infect Dis 167: 148-157.

17. Stoll BJ, Lee FK, Larsen S, Hale E, Schwartz D, et al. (1993) Clinical and serologic evaluation of neonates for congenital syphilis: a continuing diagnostic dilemma. J Infect Dis 167: 1093-1099.

18. World Health Organization (2006) Prevention of Mother and Child transmission of syphilis Safe. Integrated Management of Pregnancy and Child birth (IMPAC), World Health Organization, Geneva, Switzerland. 